\title{
Effects of Blending Sequence on Morphology and Mechanical Properties of Polypropylene/Ethylene-octene Copolymer/Clay Nanocomposites
}

\author{
Sedigheh Bagheri Kazemabad ${ }^{1, *}$, Alireza Khavandi ${ }^{1}$, Daniel Fox ${ }^{2}$, Yanhui Chen², \\ Hongzhou Zhang ${ }^{2}$, Biqiong Chen $^{3}$ \\ ${ }^{1}$ School of Metallurgy and Materials Engineering, Iran University of Science and Technology (IUST), Iran \\ ${ }^{2}$ School of Physics and CRANN, Trinity College Dublin, College Green, Ireland \\ ${ }^{3}$ Department of Materials Science and Engineering, University of Sheffield, U.K.
}

Copyright (C) 2015 by authors, all rights reserved. Authors agree that this article remains permanently open access under the terms of the Creative Commons Attribution License 4.0 International License

\begin{abstract}
The objective of this study was to investigate the effects of three blending sequences on morphology and properties of the ternary nanocomposite of polypropylene (PP)/ethylene-octene copolymer (EOC)/clay with double compatibilizers of maleated PP (PP-g-MA) and maleated EOC (EOC-g-MA) prepared by twin-screw extrusion. The $\mathrm{X}$-ray diffraction results in conjuction with transmission electron microscopy images indicated the mixture of exfoliated and intercalated structures possessed by the nanocomposite prepared by simultaneous addition of the ingredients. The nanocomposite in which clay was first mixed with PP and EOC-g-MA and then with EOC and PP-g-MA showed the same morphology. However, in the nanocomposite when clay was first mixed with PP and PP-g-MA and then mixed with EOC and EOC-g-MA, an intercalated structure was observed. The results of mechanical testing showed that there was no significant difference in the yield strength, tensile modulus and flexural modulus among the three nanocomposites. However, the Charpy impact strength of the nanocomposite prepared by simultaneous addition of ingredients was higher than that of the nanocomposites prepared in two mixing steps because of the presence of more organoclay inside the EOC phase in the former.
\end{abstract}

Keywords Polypropylene, Clay, Nanocomposite, Blending Sequence

\section{Introduction}

Nowadays, the blending of polypropylene (PP) with a rubbery phase such as ethylene-octene coplymer (EOC) is a common way to improve its impact strength [1-3]. The block copolymer of EOC is a relatively new family of polyolefin elastomers which was introduced by Dow Chemical Company in 2006 [4,5]. Adding small amounts of organoclay to the PP/EOC blends produces nanocomposites with high stiffness and reasonable impact strength $[2,3,6]$.

The effect of clay on the properties of PP/rubber blend is strongly dependent on clay location (in the matrix (PP) phase, the dispersed (rubber) phase or both phases) [7-9] as well as the dispersion degree of the clay (e.g., exfoliated, intercalated or agglomerated) [8]. The method of adding the constituents of polymer blend/clay nanocomposites to the extruder has a strong effect on the dispersion of clay and the microstructure of the nanocomposites $[7,10]$. The simplest protocol is the simultaneous addition of all components into the extruder at a temperature above the melting temperatures of both polymeric constituents. In this case if the disparity between the melting temperatures of the components is significant, clay is preferentially located in the phase that melts first. Other mixing sequences were also reported in the literature $[7,10]$. For example, the two components of the polymer blend are melted first followed by the addition of the clay. As the clay is added to both melted polymer components, clay is not in contact with any solid polymers in this case [10]. Another procedure consisted of adding clay into one polymer and then incorporating the next polymer $[7,10]$.

In the present study, Nanocomposites of $\mathrm{PP} / \mathrm{EOC} / \mathrm{maleated} \mathrm{PP}$ (PP-g-MA)/maleated EOC (EOC-g-MA)/clay were prepared by using three mixing protocols to tailor the location and dispersion of the clay in these systems and correlate these parameters to the mechanical properties. PP-g-MA and EOC-g-MA served as the double compatilizers for the nanocomposites [11]. To the authors knowledge, there has been no systematic study focusing on the mixing sequence of this type of nanocomposites. 


\section{Experimental Section}

The isotactic homopolymer of PP (PP 571P) with a melt flow index (MFI) of $5.7 \mathrm{~g} / 10 \mathrm{~min}$ at $230{ }^{\circ} \mathrm{C} / 2.16 \mathrm{~kg}$ was obtained from Saudi Basic Industries Corporation, Europe. Ethylene-octene block copolymer (Infuse ${ }^{\mathrm{TM}} 9500$ ) with a melt flow index of $5 \mathrm{~g} / 10 \mathrm{~min}\left(2.16 \mathrm{~kg}\right.$ at $\left.190^{\circ} \mathrm{C}\right)$ was obtained from Dow Chemical Company. Two compatibilizers of PP-g-MA with product number of 427845 and EOC-g-MA with trade name of Amplify ${ }^{\mathrm{TM}}$ GR216 were supplied by Sigma-Aldrich and the Dow Chemical Company, respectively. The organoclay Cloisite ${ }^{\circledR} 20 \mathrm{~A}$ (abbreviated as C20A) was provided by Southern Clay Products, Inc.

The nanocomposites of PP/EOC/PP-g-MA/EOC-g-MA/c lay were prepared using a twin screw extruder $(\mathrm{L} / \mathrm{D}=25, \mathrm{D}=$ $16 \mathrm{~mm})$. The temperature profiles were $160,160,170$ and $180^{\circ} \mathrm{C}$ and the screw speed was set at $200 \mathrm{rpm}$. The extrudates were injection molded into test specimens using a bench-top Ray-ran Test Sample Injection Moulding Apparatus. The barrel and tool temperatures were195 and $55^{\circ} \mathrm{C}$, respectively and the pressure was set at $110 \mathrm{MPa}$.

For all of the mixing sequences, the weight ratio of PP to EOC was fixed at $3: 1$. The weight $\%$ of organoclay and both the compatibilizers (ratio of PP-g-MA to EOC-g-MA $=1: 1$ ) was $4 \mathrm{phr}$ with respect to the total weight of the $\mathrm{PP} / \mathrm{EOC}$ blend. To study the effect of blending sequence on the morphology and properties of the $\mathrm{PP} / E O C / \mathrm{PP}-\mathrm{g}-\mathrm{MA} / \mathrm{EOC}-\mathrm{g}-\mathrm{MA} /$ clay nanocomposite, the following three sequences were selected: Mixture $\mathrm{P} 0$ (PP+EOC+PP-g-MA+EOC-g-MA+clay): all components were added simaltaniously to the extruder and extuded twice. Mixture P1 (PP+EOC-g-MA+clay $)+($ EOC+PP-g-MA): PP was extruded with EOC-g-MA and clay first and then EOC and PP-g-MA was added and extruded with them. Mixture P2 (PP+PP-g-MA+clay $)+($ EOC+EOC-g-MA): PP, PP-g-MA-g-MA and clay were extruded together first and afterwards EOC and EOC-g-MA were added and extruded with them. The motivation for choosing blending procedures of P1 and P2 was to locate clay mainly in PP matrix and study the dispersion state of clay particles in the matrix by using different compatibilizers.

In order to study the X-ray diffraction (XRD) of the nanocomposites, a Phillips PW 1050/80 X-ray diffractometer $(40 \mathrm{kV}, 20 \mathrm{~mA})$ was employed. The XRD was performed with a step size of $0.02^{\circ}$ in a range of $2 \theta$ from 2 to $10^{\circ}$ and a scanning time of $2.5 \mathrm{~s}$ per step. For studying the dispersion of the organoclays, specimens were sectioned by a Carl Zeiss Auriga Cross Beam focused ion beam-scanning electron microscope (FIB-SEM) workstation and evaluated by a FEI Titan 80-300 transmission electron microscope (TEM) at an acceleration voltage of $300 \mathrm{kV}$.

Tensile testing of the nanocomposites were performed using a universal Hounsfield H10KS, according to ASTM D638 and at a crosshead speed of $50 \mathrm{~mm} / \mathrm{min}$. Young's modulus was measured by employing an extensometer.
Flexural testing was measured using Instron-1011 according to ASTM D790 using 4-point bending arrangement at 2 $\mathrm{mm} / \mathrm{min}$ deformation rate. For both tensile and flexural tests, five specimens were tested. Notched Charpy impact testing of samples was carried out with a Jinjian impact tester according to BS ISO 179-1 and by using the pendulum of $1 \mathrm{~J}$. Eight specimens were performed for impact tests.

\section{Results and Discussion}

Fig.1 illustrates the XRD patterns of the organoclay (Closite 20A) and P0, P1 and P2 nanocomposites. As previously discussed $[10,11], \mathrm{C} 20 \mathrm{~A}$ presents a strong peak at around $2 \theta=3.7^{\circ}$ corresponding to a basal plane spacing $\left(\mathrm{d}_{001}\right)$ of $2.4 \mathrm{~nm}$. In mixtures P0 and P1, the diffraction peak of clay almost disappeared which may suggest the presence of some exfoliated structure in the nanocomposites. TEM analysis in our previous research [11] showed a hybrid of intercalated and exfoliated structures in mixture of P0. The relatively good dispersion of clay in this sample can be attributed to the existance of clay in both phases [11]. The XRD pattern of P1 sample is the same as P0. However, in mixture P2 the (001) peak of the clay moves to a lower angle with respect to the organoclay while maintaining a relatively high peak intensity, revealing an intercalated structure. Comparing this figure with the XRD results of our previous research [12] shows this peak is similar to the peak observed in $\mathrm{PP} / \mathrm{EOC} / \mathrm{PP}-\mathrm{g}-\mathrm{MA} / \mathrm{clay}$ nanocomposite where clay is located in PP phase, which suggests that most of the clay could be located in the PP phase as an intercalated structure in $\mathrm{P} 2$ too.

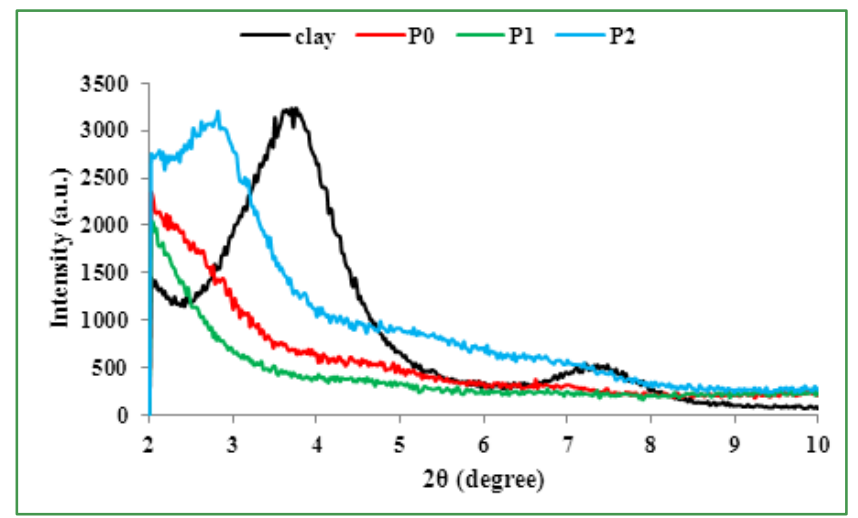

Figure 1. XRD traces of organoclay (Closite20A) [11], and the studied nanocomposites: P0 [11], P1 and P2.

The TEM images of $\mathrm{P} 1$ and $\mathrm{P} 2$ nanocomposites are shown in Fig. 2 and Fig. 3, respectively. These TEM images show that the clay layers have relatively better dispersion in the P1 than P2, with more single clay layers (indicated by green arrows) found in the nanocomposites. while most clay particles are resided in PP phase in both cases, the better dispersion state of clay in $\mathrm{P} 1$ suggests EOC-g-MA is a better compatibilizer than PP-g-MA for dispersion of clay. This 
result is in good agreement with our previous research that showed clay was dispersed better in PP/clay composite in the presence of EOC-g-MA than with PP-g-MA [13].

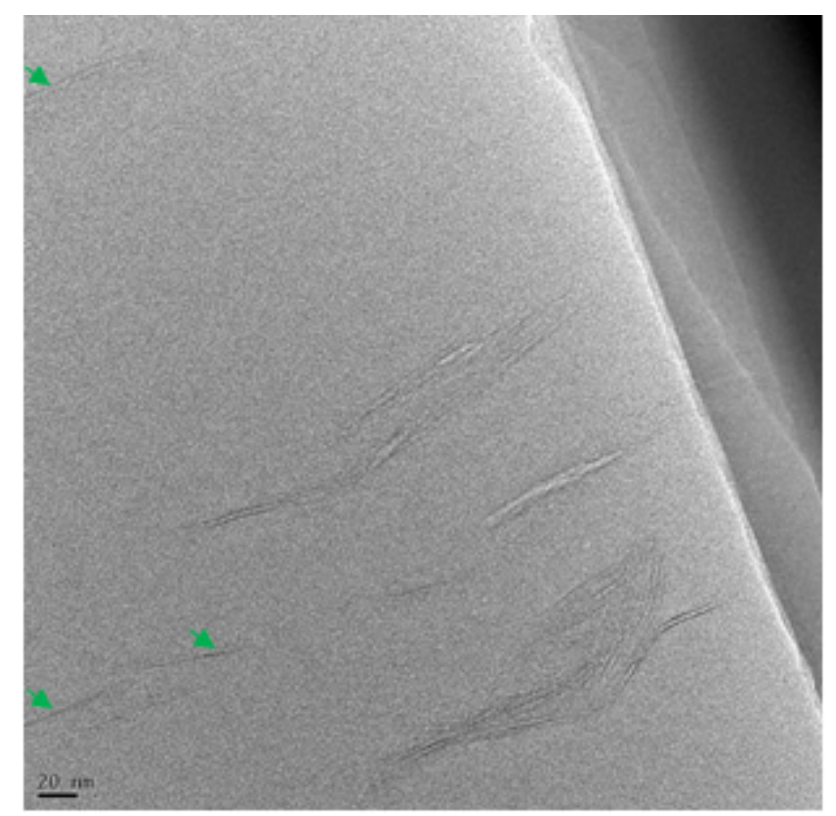

Figure 2. TEM image of P1 nanocomposite. Dark lines representing clay layers. Some clay layers are present as single exfoliated layers, indicated by the green arrows.
The results of mechanical properties of the studied mixtures are shown in Table 1. According to Table 1 there is only insignificant difference in the tensile and flexural properties of all the three nanocomposites. However, the impact strength of mixture $\mathrm{P} 0$ is higher than mixtures $\mathrm{P} 1$ and $\mathrm{P} 2$. This can be explained by the fact that in $\mathrm{P} 0$ sample, clay is dispersed in both PP and EOC phases and the presence of clay in EOC phase increases the impact strength [11]. Previous research reported that when the organoclay is located in the dispersed phase or at the interphase between the two polymers an enhancement in impact strength of polymer blends can be observed $[7,14]$. In mixtures P1 and $\mathrm{P} 2$, less clay is expected to reside in the EOC phase as clay was mixed with PP first in the presence of a compatibilizer.

Table 1. Mechanical properties of the studied nanocomposites.

\begin{tabular}{|c|c|c|c|c|}
\hline Material & $\begin{array}{c}\text { Flexural } \\
\text { Modulus } \\
(\mathrm{MPa})\end{array}$ & $\begin{array}{c}\text { Tensile } \\
\text { Modulus } \\
(\mathrm{MPa})\end{array}$ & $\begin{array}{c}\text { Tensile } \\
\text { Yield } \\
\text { Stress } \\
(\mathrm{MPa})\end{array}$ & $\begin{array}{c}\text { Impact } \\
\text { Strength }(\mathrm{kJ} / \\
\left.\mathrm{m}^{2}\right)\end{array}$ \\
\hline $\mathrm{P} 0^{\mathrm{a}}$ & $1028 \pm 18$ & $1269 \pm 130$ & $21.1 \pm 0.6$ & $9.6 \pm 3.8$ \\
\hline $\mathrm{P} 1$ & $963 \pm 16$ & $1246 \pm 73$ & $20.9 \pm 0.3$ & $6.3 \pm 2.5$ \\
\hline $\mathrm{P} 2$ & $945 \pm 35$ & $1219 \pm 59$ & $20.5 \pm 0.4$ & $7.8 \pm 4.3$ \\
\hline
\end{tabular}

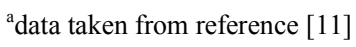

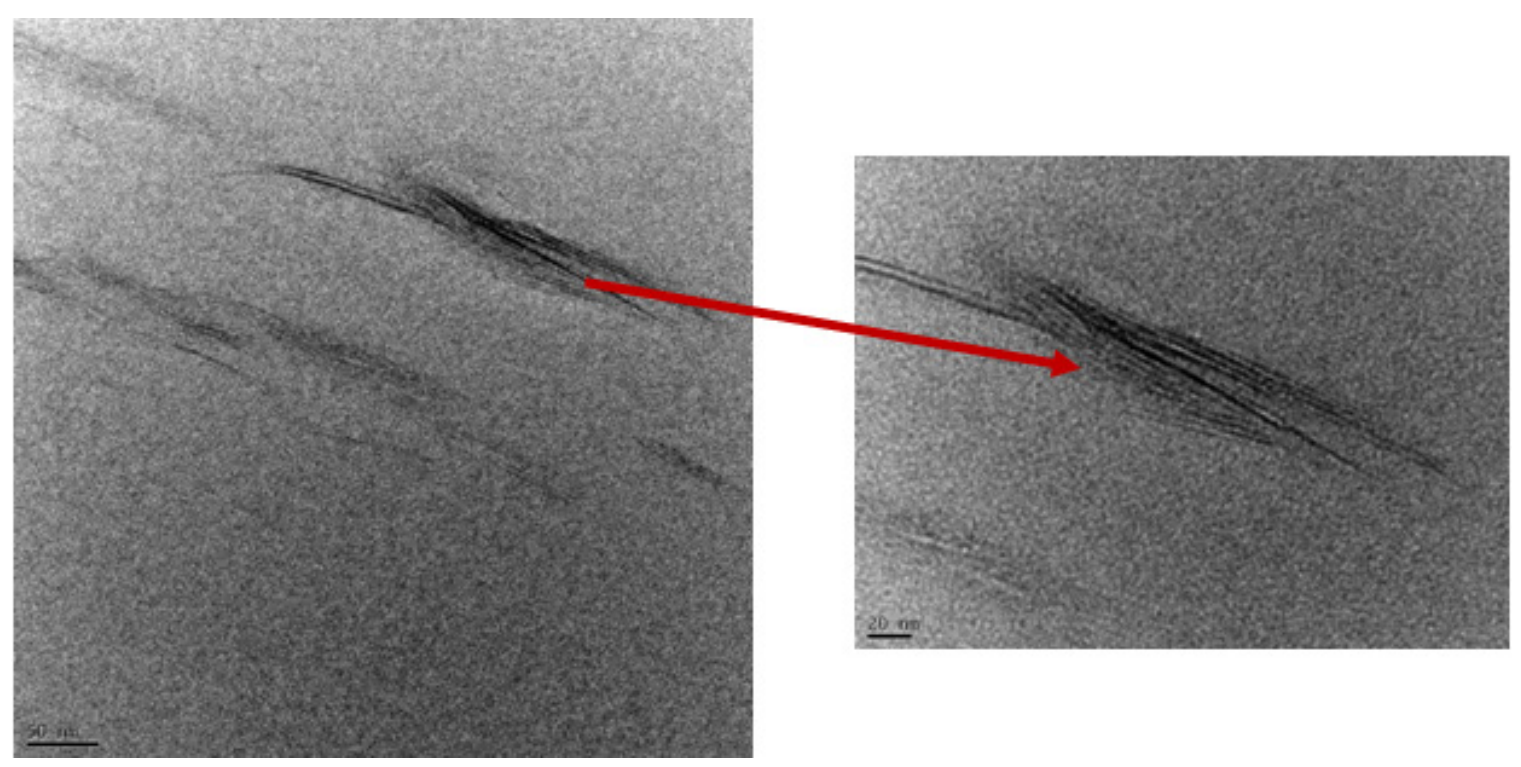

Figure 3. TEM images of $\mathrm{P} 2$ nanocomposite at different magnifications. Dark lines representing clay layers. 


\section{Conclusions}

The ternary nanocomposites of PP/EOC/clay with double compatibilizers of PP-g-MA and EOC-g-MA were prepared in three blending sequences. XRD patterns and TEM images indicated a mixture of exfoliated and intercalated structure in the nanocomposite when clay was first mixed with PP and EOC-g-MA, similar to that found for the nanocomposite prepared by simultaneous addition of all the ingredients [10]. However, in the case when clay was first mixed with PP and PP-g-MA, intercalated structure was observed. The results of mechanical properties showed stiffness and tensile strength of the three nanocomposites were nearly the same. But, the Charpy impact strength of the nanocomposite where the organoclay was dispersed in both phases of EOC and PP was higher than that of the nanocomposites where more clay was dispersed in PP phase.

\section{REFERENCES}

[1] Y. Pang, X. Dong, K. Liu, C. C.Han, E. Chen and D. Wang: Polymer Vol. 49 (2008) p. 4259

[2] D.H. Kim, P.D. Fasulo, W.R. Rodgers and D.R. Paul: Polymer Vol. 48 (2007), p. 5960

[3] H.S Lee, P.D. Fasulo, W.R. Rodgers and D.R. Paul: Polymer
Vol. 46 (2005), p. 11673

[4] P.S Chum and K.W. Swogger: Prog. Polym. Sci. Vol. 33 (2008), p. 797

[5] G. Liu, X. Zhang, C. Liu, H. Chen, K. Walton and D. Wang: Appl. Polym. Sci. Vol. 119 (2011), p. 3591

[6] F.H. Su, H.X. Huang and Y. Zhao: Composites Part B Vol. 42 (2011), p. 421

[7] C.G. Martins, N.M. Larocca, D.R. Paul and L.A. Pessan: Polymer Vol. 50 (2009), p. 1743

[8] J. R. Austin and M. Kontopoulou: Polym. Eng. Sci. (2006), p. 1491

[9] Yanmeili, G.X Wei and H.J. Sue: J. Mater. Sci. Vol. 37 (2002), p. 2447

[10] F. Fenouillot, P. Cassagnau and J.C. Majeste: Polymer Vol. 50 (2009), p. 1333

[11] S. B.Kazemabad, D. Fox, Y. Chen, H. Zhang and B. Chen: Polym. Adv. Technol. Vol. 25 (2014), p. 1116.

[12] S. B.Kazemabad, D. Fox, Y. Chen, L. M. Geever, A. Khavandi, R. Bagheri, C. L. Higginbotham, H. Zhang and B. Chen: Compos. Sci. Techno. Vol. 72 (2012), p.1697

[13] S. B.Kazemabad, A. Khavandi, D. Fox, Y. Chen, H. Zhang and B. Chen: Adv. Mater. Res. Vol. 622-623 (2013), p. 847

[14] M.U. Wahit, A. Hassan, Z.A. Mohd Ishak and T. Czigány: Express. Polym. Lett. Vol. 3 (2009), p. 309 